\title{
Incidencia del tipo de alimento sobre la sobrevivencia y ecdisis en Callinectes bocourti en cultivo para la obtención de jaiba blanda en el caribe colombiano
}

\section{Incidence of type of food on survival and ecdysis in cultured Callinectes bocourti to obtain soft crab in the Colombian Caribbean}

\author{
Germán Lozano-Beltrán*1, Erick Orozco-Acosta ${ }^{1}$, Karen Muñoz-Salas ${ }^{2}$ y \\ Klaus Rodríguez-Díaz ${ }^{1}$
}

\begin{abstract}
RESUMEN
La investigación tuvo como propósito determinar la incidencia del tipo de dieta sobre la sobrevivencia y la ecdisis en la jaiba roja (Callinectes bocourti) en cultivo, para obtener jaiba suave como alternativa de producción pesquera en el Caribe colombiano. Se realizaron 2 experimentos: 1. pescado fresco / pescado fresco $+\mathrm{Ca}^{+}$y 2. pescado fresco / alimento concentrado para camarón (34\% proteína). En 4 módulos de recirculación independiente, de 4 acuarios cada uno articulados a filtros biológicos, se sembró un ejemplar/acuario (64 jaibas por ensayo) y se realizaron 3 series de 45 días cada uno. En el experimento 1, sobrevivieron 378 ejemplares, 203 (53.7\%) correspondieron a la dieta de pescado fresco y $175(46.3 \%)$ al grupo alimentado con pescado fresco $+\mathrm{Ca}^{+}$. En el experimento 2, sobrevivieron 293 ejemplares (21.8\%), $180(61.4 \%)$ consumieron pescado fresco y $113(38.6 \%)$ alimento para camarón. En el experimento 1, no se encuentra relación entre la dieta y la ecdisis. En el experimento 2, se encuentra relación entre el sexo (hembra) y la ecdisis. Se concluye que $C$. bocourti es una especie con dificultades para manejo en cultivo y la sobrevivencia no depende de la dieta. El sexo (hembra) es una variable por considerar en la ecdisis de C. Bocourti, dado que las hembras requieren mayor energía para el proceso de maduración. La adición de $\mathrm{Ca}^{+}$al alimento natural no tiene mayor implicación en la ecdisis; puede haber mayor relación con variables ambientales y procesos hormonales que con la ingesta de $\mathrm{Ca}^{+}$.
\end{abstract}

Palabras claves: jaiba roja, jaiba blanda, ecdisis, cultivo, alimento

\begin{abstract}
The purpose of the paper was to determine the incidence of the type of diet on survival and ecdysis in cultured blunt-tooth swimming crab (Callinectes bocourti) to obtain soft crab as alternative fish production in the Colombian Caribbean. Two experiments were conducted: 1. fresh fish / fresh fish $+\mathrm{Ca}^{+}$and 2. fresh fish / shrimp concentrated feed (34\% protein). In 4 independent recirculation

1 Universidad Simón Bolívar, glozano3@unisimonbolivar.edu.co*, eorozco15@unisimonbolivar.edu.co, klausr66@ gmail.com.

2 Grupo de investigación Biología de Nutrientes, Universidad del Atlántico, bioka22@hotmail.com.
\end{abstract}


modules, each with 4 aquariums articulated to biological filters, a specimen/aquarium was planted (64 crabs per test), and 3 series were conducted of 45 days each. In experiment 1 , a total of 378 specimens survived; 203 (53.7\%) had the fresh fish diet, while $175(46.3 \%)$ were fed fresh fish $+\mathrm{Ca}^{+}$. In experiment 2, a total of $293(21.8 \%)$ specimens survived; 180 (61.4\%) consumed fresh fish and $113(38.6 \%)$ shrimp feed. There is no relationship between diet and ecdysis in experiment 1 , while there is relationship between sex (female) and ecdysis in experiment 2 . It is concluded that $C$. bocourti is a species with crop management difficulties, and survival does not depend on diet. Sex (female) is a variable to consider in the ecdysis of $C$. bocourti since females require more energy for the maturation process. Adding $\mathrm{Ca}^{+}$to natural food has no greater implications in ecdysis. There may be a stronger relationship with environmental variables and hormonal processes than with the $\mathrm{Ca}+$ intake.

Keywords: blunt-tooth swimming crab, soft shell crab, ecdysis, culture, food

\section{INTRODUCCION}

Los cangrejos del género Callinectes (Crustacea: Portunidae) son decápodos nadadores que se encuentran ampliamente distribuidos en ambientes tropicales y subtropicales en el océano Atlántico, el mar Caribe y el océano Pacífico (Williams, 1974). En el Caribe colombiano, su pesquería tiene importantes implicaciones de orden socioeconómico y el esfuerzo pesquero se ha centrado en la Ciénaga Grande de Santa Marta (Lasso-Guzmán \& Ordoñez-Pardo, 1987; Valencia-Cuellar, 1994; Valencia-Cuellar \& CamposCampos, 1994; Correa-Daza, 2002; Lozano-Beltrán \& De León-Martínez, 2008). Al igual que muchos de los organismos acuáticos, las poblaciones de especies del género Callinectes han disminuido drásticamente, debido a múltiples causas como el deterioro de su hábitat por acción antrópica, procesos naturales (Sánchez-Páez et al. 1997), la sobrepesca y la falta de ordenación de su pesquería. En este sentido, los informes del Instituto de Investigaciones Marinas y Costeras (INVEMAR) en la Ciénaga Grande de Santa Marta, sobre la pesquería de jaiba, indican que las poblaciones naturales se encuentran deterioradas y recomiendan atención oportuna antes de que colapsen (Ibarra et al. 2014). La acuicultura ha surgido como una alternativa productiva y la jaiba blanda (soft shel lcrab) se vislumbra como una posibilidad viable en las zonas costeras colombianas, mediante el cultivo en sistemas de recirculación de agua, lo que implica mejoramiento de la productividad y aumento en la competitividad a la industria jaibera, basada desde hace más de 50 años en la extracción de los 5 tipos de carnes que se obtienen del proceso tradicional en planta (Jiménez et al. 2002; Morillo, 2006). En Estados Unidos, la jaiba blanda es un producto que se ha consumido desde hace más de 100 años. Los registros señalan que, durante mucho tiempo, el origen de la jaiba blanda fue el medio natural, mediante 
la colecta de ejemplares recién mudados (Osterling, 1995; Stagg \& Whilden, 1997). A mediados de los años 1800 , se iniciaron las actividades de cultivo, colectando ejemplares en etapa de preecdisis, hasta la finalización del proceso de ecdisis en sistemas controlados. En la actualidad, se investiga sobre la regulación endocrina del proceso de ecdisis, la acción de la hormona inhibidora de la ecdisis y los niveles hormonales durante este, en especies del género Callinectes y en otros crustáceos como Gecarcinus lateralis y Homarus americanus (Lee et al. 1998; Zheng-Junying et al. 2006; Chang \& Mykles, 2011).

En México, la primera planta para producción de jaiba suave se instaló en el puerto de Veracruz y, posteriormente, en Tamaulipas y Campeche, en 1985. Luego, en 1993, se instaló una planta en Ciudad del Carmen y, tras su cierre, se abrió un centro experimental en el Centro de Estudios Tecnológicos del Mar \# 29 (estado de Campeche), reiniciado como proyecto de formación y de producción en el 2004 (Maya de la Cruz et al. 2007; Chan, 2011). En el Pacífico mexicano, Pérez-González (2005) evaluó la influencia del alimento en la ecdisis en $C$. bellicosus y Vega-Villasante et al. (2006) desarrollaron investigación con $C$. arcuatus, mediante la que produjeron un manual descriptivo, adaptando las técnicas de producción a la biología y circunstancias propias tanto de la especie como del ambiente. $\mathrm{La}$ producción de jaiba suave se presenta como opción productiva con la idea de diversificar y posibilitar un valor agregado a la producción de jaiba, poniendo de presente las implicaciones de orden social de su pesquería (Schleske-Morales, 2008; Ibarra et al. 2015), ante la necesidad de proteger el recurso natural de factores como la sobrepesca, la degradación ambiental y la carencia de normas para la pesquería.

En Cuba, se investigó el cultivo de jaiba azul C. sapidus, en la búsqueda de un manejo que permitiera garantizar el aprovechamiento responsable de las especies de jaiba en su medio natural, al seleccionar únicamente los individuos próximos a la ecdisis (Reyes et al. 2009; Reyes-Canino et al. 2003). En este sentido, se establecieron las bases técnicas para su cultivo, tales como el manejo previo a la estabulación de los ejemplares y la identificación de las señales de pre-ecdisis y ecdisis (Siam \& Castelo, 2011).

En condiciones naturales, las jaibas buscan el alimento en la oferta del medio y el pescado constituye la base de su dieta, razón por la cual los pescadores utilizan diferentes especies de mugilidos como carnada en las nasas jaiberas. El cultivo comercial de otros crustáceos, como camarones y langostinos (Pennaeus sp.), se ha alcanzado con éxito, en términos de sobrevivencia y crecimiento, mediante la alimentación con concentrados comerciales para camarón. El $\mathrm{CaCO}_{3}$ es el 
principal componente del caparazón de las jaibas, el cual se deposita naturalmente hasta llegar a constituirse en un importante elemento de su exoesqueleto. Por estas razones, se consideró pertinente determinar, en términos de sobrevivencia y proceso de ecdisis, la incidencia del tipo de alimento suministrado a las jaibas en cultivo, evaluando el efecto del alimento concentrado para camarón y la adición de calcio a la dieta natural.

\section{MATERIALES Y MÉTODOS}

Área de estudio: El estudio se llevó a cabo entre agosto de 2015 y febrero de 2016, en las instalaciones que la Universidad Simón Bolívar de Barranquilla adaptó para los propósitos de la investigación, en inmediaciones del puente de $\mathrm{La}$ Barra, aledaño a la Ciénaga Grande de Santa Marta, municipio de Pueblo Nuevo (Magdalena, Colombia).

Montaje: Se trabajó un prototipo modular (Fig. 1), conservando el concepto de reutilización constante de agua, manteniendo en equilibrio los parámetros fisicoquímicos en el sistema (oxígeno disuelto, temperatura y salinidad), iniciando por el componente del tratamiento de agua que llega de las unidades de cultivo, la cual fue sometida a diversos pasos con retorno al sistema de cultivo con la calidad adecuada, para sostener los rangos vitales de confort que precisan los ejemplares.

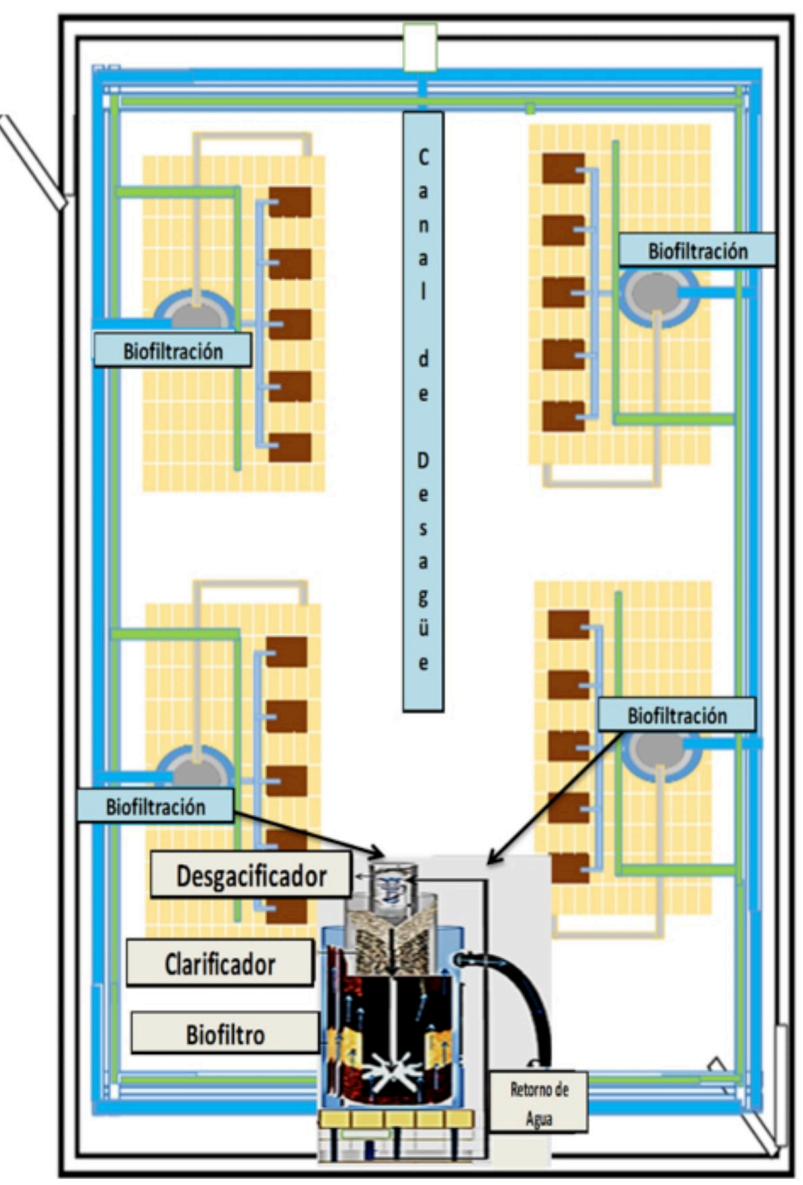

Fig. 1. Esquema del prototipo modular empleado para el cultivo de C. bocourti en el Caribe colombiano Fig. 1. Scheme of the modular prototype used for the culture of $C$. bocourti in the Colombian Caribbean

Se montaron 4 unidades de 16 acuarios cada una (3 $600 \mathrm{~cm}^{3}$ de volumen operacional de agua por unidad), articuladas a un sistema de biofiltración, el cual consistió en un recipiente cilíndrico plástico de $900 \mathrm{~L}$ de capacidad 
total, con los mantos filtrantes en el interior y el material necesario para la fijación de bacterias encargadas del desdoble de los compuestos nitrogenados (controlar el nitrógeno amoniacal total NAT), integrando así los tratamientos físicos y químicos.

Para la remoción y/o retención de sólidos, se instaló un clarificador $(25 \mathrm{~L}$ de capacidad total), donde se recibió el agua con los sólidos sedimentables que fueron removidos, mediante filtración mecánica con medio granular expandible. Una vez retenido el material solido más grueso e incluso buena parte del fino, el agua retornó al biofiltro por una tubería de 1 " de diámetro. Como complemento del biofiltro, se instaló un desgasificador, cuya función era retirar los excesos de $\mathrm{CO}_{2}$ del sistema.

Se realizaron 3 ciclos de producción experimental. Fueron estabuladas igual número de jaibas (64), durante los 135 días que duró la experimentación ( 3 ciclos de 45 días cada una); el montaje de los módulos se mantuvo a lo largo de 6 meses, durante los cuales transcurrieron las pruebas. El oxígeno de cada sistema de cultivo fue suministrado por aireadores de motores eléctricos dispuestos para cada módulo de manera independiente. Antes de cada inicio de ciclo, se desinfectaron las líneas de aire, utilizando agua con alcohol (70\%), y los 16 acuarios se lavaron con cepillo y agua. Cada unidad de trabajo fue curada con agua dulce cruda durante 8 días, se lavó con agua dulce tratada y filtrada, se desinfectó (detergente, enjuague de cloro líquido a $5 \mathrm{pp}$, película líquida de Iodopovidona al $1 \%$ ) y se enjuagó de nuevo con agua dulce tratada y filtrada. Posteriormente, se adicionó solución de peróxido de hidrógeno (20\%), se dejó reposar por $30 \mathrm{~min}$, para finalmente enjuagar con solución de Iodopovidona (1\%) y lavar con agua dulce.

Se inició el llenado de los módulos con agua de mar de los tanques reservorios, con una salinidad entre 3 y 20 PSU, debido a la variabilidad estacional por efecto de las lluvias in situ en el transcurso de las pruebas. Se filtró el agua de mar con filtros tipo bolsos de una micra de poro y se dejó circular el agua en el sistema, con calibración constante de flujo y aireación permanente. Una vez cumplidos el llenado y la estabilización de flujos, se adicionó Melaza (40\% fuente de carbono) como potenciador de las bacterias del biofiltro. Vale reseñar que los biofiltros de cada módulo quedaron en recirculación, durante los 6 meses sin interrupción, independientemente de los vacíos entre prueba y prueba.

Colecta y transporte del material biológico: Previa a la llegada de las jaibas a la planta de cultivo, procedentes de la pesca nocturna en el medio natural, se hizo una revisión final del flujo de entrada y salida de agua de cada acuario, de la aireación y de su paso por el sistema de recirculación, 
así como lecturas de las condiciones físicas y químicas del medio.

El material biológico procedente de capturas de pesca artesanal de la Ciénaga Grande de Santa Marta fue seleccionado uno a uno (cada ejemplar), sin separar por sexo, observando las señales de ecdisis que se especificaron para este procedimiento, de acuerdo con los criterios señalados por Lozano-Beltrán et al. (2015).

El traslado a la sala de la planta de cultivos se efectuó en canastas plásticas cubiertas de tela gruesa, humedecida con agua de mar para dar oscuridad y mantener la humedad del medio, con lo que se obtuvo disminución de la agresividad de los ejemplares y total sobrevivencia. Una vez en la planta, se trasladaron a tanques de 500 L (tipo bebedero), en donde se realizó el cultivo masivo, que fue básicamente la adaptación a las condiciones controladas, proceso que sirvió para acercar las jaibas a las condiciones físico-químicas del cultivo con el menor estrés y la mínima mortalidad posibles.

Se realizó una revisión fenotípica y la verificación del estado de pre-ecdisis de cada individuo. Se llevó a cabo la biometría, con el fin de asegurar que cada ejemplar entraba al cultivo con una talla superior a los $8.5 \mathrm{~cm}$ de Abel (abertura entre las espinas laterales del caparazón) (Fig. 2), tamaño adecuado para su mercadeo como jaiba suave. Una vez concluido el proceso de adaptación a las condiciones de cultivo, se dio inicio a los experimentos, con el propósito de evaluar la incidencia del alimento en la ecdisis y sobrevivencia en cultivo de $C$. bocourti.

Las jaibas estabuladas fueron observadas durante 12 horas, para determinar mortalidad asociada a la captura y el transporte de las jaibas, desde el sitio de captura hasta la planta de cultivos. Finalmente, se comenzaron los experimentos.

El experimento 1 consistió en alimentación con pescado fresco (Mugil incilis) y alimento concentrado para camarón (34\% de proteína), con la variante de la adición de calcio mineral $\left(\mathrm{Ca}^{+}\right)$al pescado fresco. El pescado fue fileteado y cortado en cubos de aproximadamente $1 / 2 \mathrm{~cm}^{3}$. El calcio empleado para el experimento

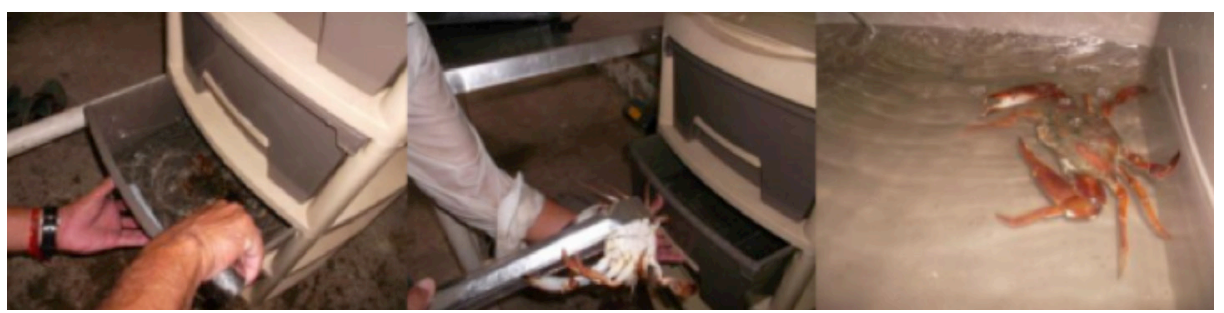

Fig. 2. Selección de jaibas para prueba preliminar

Fig. 2. Crab selection for preliminary test 
fue de una casa comercial $\left(\mathrm{Ca}^{+}-600\right.$ $\mathrm{mg}$ de calcio elemental). Fue fragmentado, pulverizado y agregado a cada porción de pescado fresco, mediante adición por acción envolvente de la porción y secado al ambiente, asi como suministrado a saciedad 3 veces por día.

Se emplearon 256 ejemplares / ciclo. Se seleccionó un total de 1537 ejemplares de C. bocourti y se evaluó alimento natural (pescado fresco) / pescado fresco $+\mathrm{Ca}^{+}$.

En el experimento 2, se seleccionaron 1344 ejemplares de C. bocourti y se evaluó alimento natural / alimento concentrado para camarón. Se suministró alimento a saciedad y la frecuencia fue 3 veces por día.

La verificación del inicio de ecdisis se realizó revisando cada acuario 3 veces por día y se observó si había señales del inicio del proceso, de acuerdo con el criterio de Vega-Villasante et al. (2006) y la observación de señales como la ruptura de la línea de sutura ventral, un leve levantamiento de la parte posterior del caparazón y la disminución en la ingesta de alimento o ayuno total.

Las variables fisico-químicas se registraron cada 6 horas, en los horarios de 6 a. m., 12 m., 6 p. m. y 12 a. m., en los acuarios. Las lecturas de temperatura y oxígeno disuelto se realizaron con un equipo multiparámetros marca YSI (modelo 550 A) y la salinidad, con un refractómetro marca Premium (modelo SR6).

Para cada experimento, se determinó el número de jaibas que sobrevivieron durante el cultivo y las que culminaron el proceso de ecdisis. Además, se estimó el riesgo asociado a las diferentes dietas.

Análisis estadístico: Se analizó la normalidad de los datos y, posteriormente, se aplicó estadística descriptiva (media, moda, frecuencia, porcentajes). Se realizaron tablas de contingencia y se aplicó el modelo de regresión para las pruebas de alimentación, teniendo como objetivo establecer la sobrevivencia como variable categórica, en función de la dieta (pescado fresco, pescado fresco $+\mathrm{Ca}^{+}$y alimento concentrado). Además, se estimaron los riesgos y se calcularon los intervalos de confianza (95\%), para determinar el riesgo asociado a las dietas y la sobrevivencia, durante el proceso de cultivo en C. bocourti, en los experimentos 1 y 2 , mediante RR. Se aplicó la prueba de $X^{2}$ de Pearson (P-Valor), para establecer si existe diferencia estadísticamente significativa entre el tipo de dieta sobre sobrevivencia y la ecdisis.

Se empleó el modelo de regresión binario para la sobrevivencia, en función de variables como sexo y ecdisis. Para los parámetros fisico-químicos, se utilizó el análisis descriptivo (media, mediana, moda, desviación estándar, mínimos y máximos). 


\section{RESULTADOS}

La evaluación de la incidencia de la dieta en la sobrevivencia en el cultivo de $C$. bocourti muestra que en el experimento 1 sobrevivieron 378 ejemplares (24.6\%), de los cuales 203 (53.7\%) correspondían a la dieta de pescado fresco y $175(46.3 \%)$ a la de pescado fresco $+\mathrm{Ca}^{+}$. En el experimento 2, sobrevivieron 293 (21.8\%) individuos, de los cuales 180 (61.4\%) consumieron pescado fresco y 113 (38.6\%) recibieron alimento concentrado para camarón, evidenciando que, en esta etapa del experimento, la sobrevivencia no depende de la dieta, al no encontrar diferencias estadísticamente significativas $(P \geq 0.05)$ (Cuadro 1).
En el experimento 1, en el grupo que recibió la dieta de pescado fresco, la sobrevivencia fue de 102 (53.1\%) para hembras y $101(54.3 \%)$ para machos. Por otro lado, en el grupo que fue alimentado con pescado fresco + $\mathrm{Ca}^{+}$, la sobrevivencia fue 90 (46.9\%) para hembras y $85(45.7 \%)$ para machos, lo que indica que esta fue similar en los dos sexos en el experimento 1 , al no encontrarse diferencias estadísticamente significativas $\left(x^{2}=0.53\right.$; $P=0.50)$.

En el experimento 2, el grupo que recibió la dieta de pescado fresco presentó una sobrevivencia de 82 hembras $(53.6 \%)$ y 71 machos $(46.4 \%)$ y, en el fue alimentado con concentrado para camarón, sobrevivieron 91 hembras $(65.5 \%)$ y 49 machos $(34.5 \%)$. Se encontró diferencias estadísticamente

Cuadro 1. Sobrevivencia de C. bocourti. Experimento 1: pescado fresco / pescado fresco $+\mathrm{Ca}^{+}$y experimento 2: pescado fresco / concentrado para camarón Table 1. Survival of C. bocourti. Experiment 1: fresh fish / fresh fish $+\mathrm{Ca}^{+}$and experiment 2: fresh fish/shrimp concentrate

\begin{tabular}{cccc}
\hline Experimentos & Dieta & $\begin{array}{c}\text { Sobrevivencia } \\
\mathbf{n}(\mathbf{\%})\end{array}$ & $\mathbf{X}^{2}$ (P-Valor) \\
\hline \multirow{2}{*}{1} & Pescado fresco & $203(53.7 \%)$ & $2.70(0.10)$ \\
& Pescado fresco $+\mathrm{Ca}^{+}$ & $175(46.3 \%)$ & \\
Total & 378 & $0.395(0.5)$ \\
\hline \multirow{2}{*}{2} & Concentrado para camarón & $140(21.1 \%)$ & \\
& Pescado fresco & $153(22.5 \%)$ & \\
\hline
\end{tabular}

Prueba de chi cuadrado $\left(\mathrm{X}^{2}\right)$ con un grado de libertad, $P \leq 0.05$ indican diferencias estadísticamente significativas. 
significativas entre el sexo y la sobrevivencia en el experimento $2\left(x^{2}=\right.$ 3.93; $P=0.05$ ) (Cuadro 2).

En relación con el proceso de ecdisis, para el experimento 1, en la dieta de pescado fresco, en los machos se presentó ecdisis en el 48.6\% (53 ejemplares) y en las hembras en el $52.1 \%$ (63 ejemplares). En la dieta pescado fresco $+\mathrm{Ca}^{+}$, en los machos la ecdisis se presentó en el 51.4\% (56 ejemplares) y en las hembras en el $47.9 \%$ (58 ejemplares). Para el experimento 2, la ecdisis en los machos fue de $42.2 \%$ (38 ejemplares) y en las hembras alimentadas con pescado fresco de 57.8\% (52 ejemplares). En tanto en las hembras con la dieta de alimento concentrado fue de $65.5 \%$ (57 ejemplares) y en los machos de $34.5 \%$ (30 ejemplares), no se encontró diferencias estadísticamente significativas según el sexo, entre las dietas y la ecdisis (Cuadro 3). $\mathrm{Al}$ vincular el riesgo relativo entre las dietas experimentadas y la sobrevivencia en los procesos de cultivo de $C$. bocourti, se evidencia que no hay asociación. Para el experimento 1 el riesgo relativo fue $\mathrm{RR}=0.82$ y para el experimento 2 de $R R=1.073$ (Cuadros 4 y 5). De esta manera, se encuentra que la sobrevivencia de $C$. bocourti no guarda relación con el tipo de dieta empleada, aunque esta puede ser un factor protector para la sobrevivencia.

El modelo de regresión binaria evidencia que la dieta de pescado fresco / pescado fresco $+\mathrm{Ca}^{+}$del experimento $1 \mathrm{y}$ la ecdisis no se encuentran relacionadas (Cuadro 6). En

Cuadro 2. Comparación estadística entre la sobrevivencia, las dietas y el sexo de $C$. bocourti

Table 2. Statistical comparison between survival, diets, and sex of $C$. bocourti

\section{Sobrevivencia a los procesos de cultivo}

\begin{tabular}{cccccc} 
Sexo & \multicolumn{2}{c}{ Dieta de pescado fresco } & \multicolumn{3}{c}{ Dieta de pescado fresco $+\mathbf{C a}^{+}$} \\
& $\mathbf{n}$ & $\mathbf{\%}$ & $\mathbf{n}$ & $\mathbf{\%}$ & $\mathbf{X}^{2}$ (P-Valor) \\
\hline Hembras & 102 & 53.1 & 90 & 46.9 & $0.53(0.50)$ \\
Machos & 101 & 54.3 & 85 & 45.7 & $3.93(0.05)^{*}$ \\
\hline \multicolumn{3}{c}{ Dieta de pescado fresco } & \multicolumn{5}{c}{ Al. concentrado para cama- } \\
Hembras & 82 & 53.6 & 91 & 65.5 & 3.5 \\
Machos & 71 & 46.4 & 49 & 34.5 & \\
\hline
\end{tabular}

Prueba de chi cuadrado $\left(\mathrm{X}^{2}\right)$ con un grado de libertad, $P \leq 0.05$ indica diferencias estadísticamente significativas. 
Cuadro 3. Comparación estadística entre la ecdisis, las dietas y el sexo de $C$. bocourti

Table 3. Statistical comparison between ecdysis, diets, and sex of C. bocourti

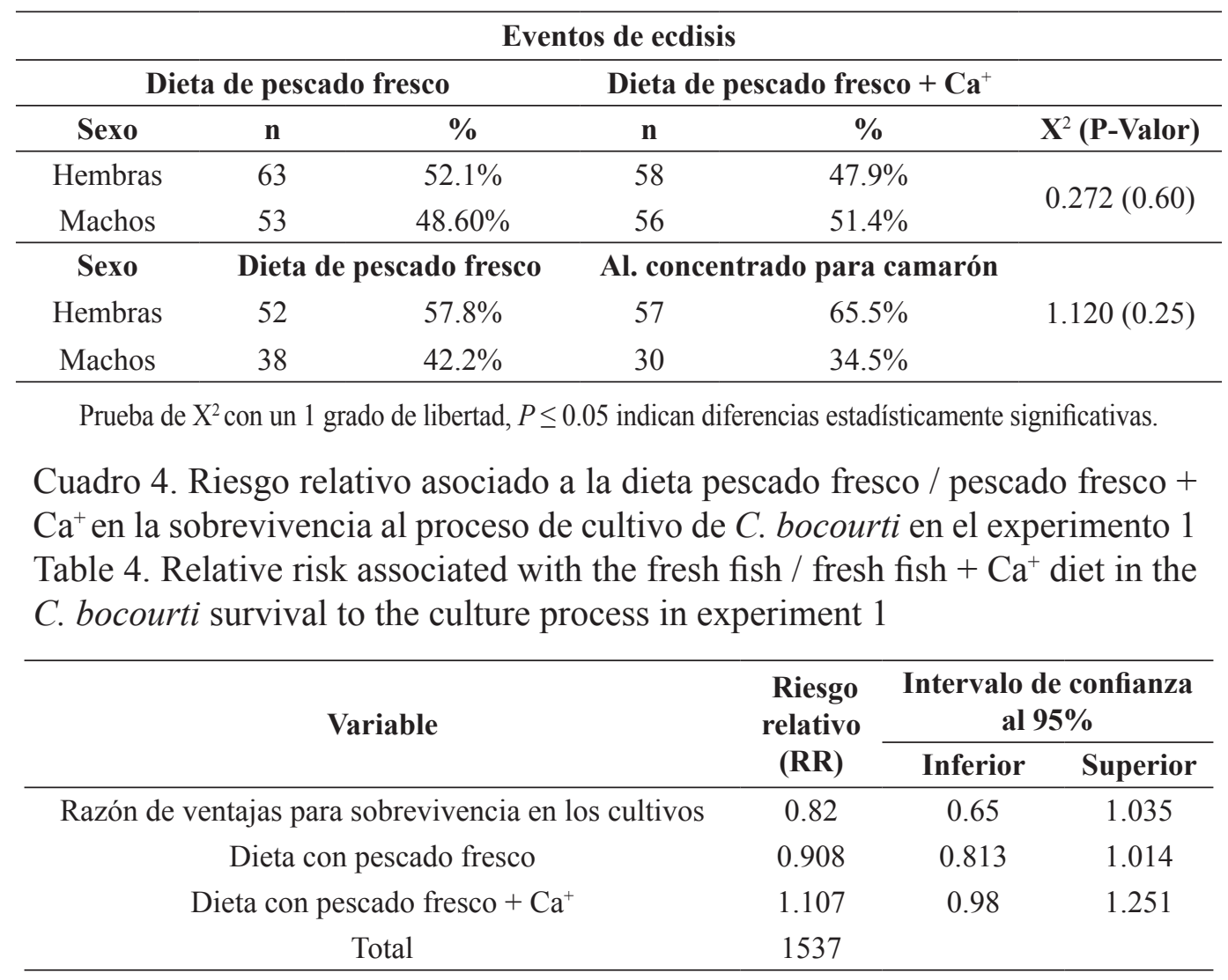

Cuadro 5. Riesgo relativo asociado a la dieta de pescado fresco / alimento concentrado para camarón en la sobrevivencia al proceso de cultivo de $C$. bocourti en el experimento 2

Table 5. Relative risk associated with the fresh fish / shrimp concentrated feed diet in the $C$. bocourti survival to the culture process in experiment 2

\begin{tabular}{cccc}
\hline Variables & $\begin{array}{c}\text { Riesgo rela- } \\
\text { tivo (RR) }\end{array}$ & \multicolumn{2}{c}{ Intervalo de confianza de 95\% } \\
\cline { 4 - 4 } & 1.073 & 0.823 & Inferior \\
\hline Razón de ventajas para dieta (concentrado & & 0.959 & 1.399 \\
con camarón / pescado fresco) & 1.015 & 0.768 & 1.075 \\
No presentaron sobrevivencia & 0.946 & & 1.166 \\
Sí presentaron sobrevivencia & 1344 & & \\
Total & &
\end{tabular}


el experimento 2, la dieta de pescado fresco y el alimento concentrado para camarón se relacionan con la ecdisis (Cuadro 7).
Los parámetros fisicoquímicos registrados durante el periodo de cultivo, en los experimentos, fueron oxígeno, temperatura y salinidad (Cuadro 8).

Cuadro 6. Modelo de regresión binario para la sobrevivencia en el cultivo, la ecdisis y el sexo de C. bocourti en el experimento 1

Table 6. Binary regression model for $C$. bocourti culture survival, ecdysis, and sex in experiment 1

Modelo de regresión binaria para el experimento 1

95\% C. I. para

$\operatorname{EXP}(B)$

\begin{tabular}{ccccccccc}
\hline Variable & $\mathbf{B}$ & $\begin{array}{c}\text { Error } \\
\text { estándar }\end{array}$ & Wald & gl & Sig. & $\operatorname{Exp(B)}$ & Inferior & Superior \\
Ecdisis & -0.267 & 0.197 & 1.827 & 1 & 0.176 & 0.766 & 0.52 & 1.280 \\
Sobrevivencia & 0.346 & 0.0164 & 4.437 & 1 & 0.035 & 1.413 & 1.024 & 1.949 \\
Sexo & -0.049 & 0.102 & 0.225 & 1 & 0.0635 & 0.953 & 0.779 & 1.164 \\
Constante & -0.042 & 0.312 & 0.019 & 1 & 0.892 & 0.958 & & \\
\hline
\end{tabular}

No hay relación estadística entre la ecdisis y el sexo de C. bocourti en el experimento 1 . Si hay relación estadística, pero se presenta de riesgo con la sobrevivencia del experimento 1. Según los exp. b e intervalos.

Cuadro 7. Modelo de regresión binario para la sobrevivencia en el cultivo, la ecdisis y el sexo de C. bocourti en el experimento 2

Table 7. Binary regression model for $C$. bocourti culture survival, ecdysis, and sex in experiment 2

Modelo de regresión binaria para el experimento 2

95\% C. I. para

EXP (B)

\begin{tabular}{ccccccccc}
\hline Variables & B & $\begin{array}{c}\text { Error es- } \\
\text { tándar }\end{array}$ & Wald & gl & Sig. & Exp (B) & Inferior & Superior \\
Ecdisis & -0.097 & 0.216 & 0.201 & 1 & 0.654 & 0.908 & 0.595 & 1.386 \\
Sobrevivencia & 0.132 & 0.177 & 0.556 & 1 & 0.456 & 1.141 & 0.806 & 1.615 \\
Sexo & -0.061 & 0.114 & 0.288 & 1 & 0.592 & 0.941 & 0.753 & 1.176 \\
Constante & 0.005 & 0.35 & 0 & 1 & 0.989 & 1.005 & & \\
\hline
\end{tabular}

Existe asociación significativa entre la ecdisis y el experimento 2 (es de protección). No hay asociación significativa en entre el sexo y el experimento 2 . No hay asociación significativa entre sobrevivencia y el experimento 2 . 
Cuadro 8. Parámetros fisicoquímicos registrados en el cultivo de los experimentos 1 y 2

Table 8. Physicochemical parameters recorded in culture of experiments 1 and 2

\begin{tabular}{ccccc}
\hline \multirow{2}{*}{ Experimentos/dieta } & Medidas & $\begin{array}{c}\text { Pxígeno } \\
\text { disuelto } \\
(\mathbf{m g} / \mathbf{L})\end{array}$ & $\begin{array}{c}\text { Temperatura } \\
\left({ }^{\circ} \mathbf{C}\right)\end{array}$ & $\begin{array}{c}\text { Salinidad } \\
\text { (PSU) }\end{array}$ \\
\hline \multirow{2}{*}{1 (Pescado fresco + pesca- } & Media & 3.04 & 23.84 & 9.75 \\
do fresco $\left.+\mathrm{Ca}^{+}\right)$ & Mediana & 3.31 & 24 & 9 \\
& Moda & 3.3 & 24 & 9 \\
& Desviación estándar & 0.81 & 1.06 & 1.91 \\
2 (Pescado fresco + & Mínimo & 0.5 & 16 & 6 \\
alimento concentrado para & Máximo & 6.3 & 26.5 & 20 \\
camarón) & Media & 3.32 & 23.74 & 9.47 \\
& Mediana & 3.31 & 24 & 9 \\
& Moda & 3.3 & 24 & 9 \\
& Mínimo & 0.5 & 16 & 1915 \\
& Máximo & 6.3 & 26.5 & 6 \\
& & & & 20 \\
\hline
\end{tabular}

\section{DISCUSIÓN}

Esta es la primera investigación del cultivo de $C$. bocourti que se realiza en el continente americano para la obtención de jaiba blanda o suave (soft shell crab), ya que los experimentos realizados en otros países, como Cuba, México y Estados Unidos, se han centrado en las especies C. rathbune, $C$. sapidus, C. arcualtus y $C$. bellicosus. La baja sobrevivencia (23.2\%) en los 2 experimentos muestra que C. bocour$t i$ es una especie poco adaptable a las condiciones de cultivo de estos y, consecuentemente, dificulta las labores de cultivo y seguimiento de los efectos de las variables estudiadas, como la adición de calcio y la alimentación con el concentrado para camarón. Aunque en las investigaciones revisadas no se evalúa la sobrevivencia de los ejemplares, en la presente investigación se encontró que los ejemplares alimentados con pescado fresco mostraron una mayor sobrevivencia durante todo el proceso de cultivo.

Entre los trabajos realizados para la obtención de jaiba suave en condiciones controladas, se identifican los métodos de intervención física (quelotomía uni o bilateral y ablación ocular), 
manejo del alimento y modulación de factores ambientales, como elementos de incidencia directa, tanto en el proceso de ecdisis, como en la disminución del tiempo de inter-ecdisis, en investigaciones adelantadas con C. sapidus y C. rathbune (Ary Jr. et al. 1987; Amador del Ángel et al. 1992; Amador del Ángel et al. 1996; Ibarra-Silva \& Santamaría-Gallegos, 1993).

Pérez-González (2005), realizó investigación sobre la influencia del alimento en la ecdisis en C. bellicosus, empleando 3 tipos de dieta, alimento natural (almeja + filete de pescado) con un nivel de $65 \%$ de proteína y 2 alimentos concentrados para camarón (35\% y $40 \%$ de proteína). Al igual que en el presente estudio, en la evaluación de las dietas hallaron que el alimento natural es el más adecuado para el cultivo de las jaibas, al haber diferencias significativas en el tiempo de inter ecdisis $(P<0.05)$. En cuanto a la evaluación de los alimentos concentrados, se evidencia que el mejor resultado se obtuvo con el de $40 \%$ de proteína. Para el caso de $C$. bocourti, aunque acepta el alimento concentrado para camarón (34\% de proteína), hay preferencia por el pescado fresco, probablemente debido a que, siendo este su alimento natural, presenta mayor atractabilidad para los ejemplares confinados.

El éxito en obtención de la jaiba suave radica en la buena selección de ejemplares, en especial, en la observación de las señales de ecdisis, mas no se hace propuesta específica para el manejo de la alimentación durante el proceso de cautiverio (Reyes-Canino et al. 2003; Siam \& Castelo, 2011). Sin embargo, estos autores no hacen propuestas específicas para el manejo de la alimentación durante el proceso de cautiverio. De igual manera, Cortés-Jacinto \&Vega-Villasante (2009) resaltan que las señales de ecdisis en C. arcuatus son claves para el éxito del proceso dirigido a la obtención de jaiba suave.

En relación con el agregado de calcio al alimento natural, no parece haber incidencia con la disminución del tiempo de intermuda o con los eventos de ecdisis, dado que esta es un proceso natural en todos los crustáceos y podría estar más asociado a la secreción de hormonas como lo mencionan Lee et al. (1998).

En el presente estudio, se encontró que la dieta de pescado fresco y alimento concentrado para camarón guarda relación con el sexo (hembra) y la ecdisis, lo cual podría explicarse por el hecho de que las hembras necesitan mayor cantidad de energía que los machos, en los procesos de maduración sexual, requieren cantidad de glucógeno, lípidos y proteínas para la culminación de este proceso, así como para la generación del nuevo caparazón, eventos extremadamente costosos en términos de energía (Fernández-Luna, 1998; Fernández-Luna et al. 1999; Vega-Villasante et al. 1999; Charron et 
al. 2014). En este estudio, el consumo de pescado fresco y de alimento concentrado para camarón por parte de C. bocourti, posiblemente, cubre las necesidades energéticas, por su alta calidad nutricional.

\section{CONCLUSIÓN}

La sobrevivencia a las condiciones de cultivo muestra que $C$. bocourti es una especie con dificultades para manejo en condiciones controladas, como es evidente en la baja sobrevivencia que mostraron los diferentes experimentos.

La sobrevivencia de $C$. bocourti durante el cultivo no dependió de la dieta, mas si parece que la dieta es un factor protector; es decir, ejemplares con una buena dieta presentan un menor riesgo de mortalidad.

Los resultados y la evaluación estadística demuestran que, posiblemente, el sexo juega un papel importante en la ecdisis de C. Bocourti; adicionalmente, evidencian que agregar $\mathrm{Ca}^{+}$al alimento natural no tiene mayor implicación en el proceso de ecdisis y pueden estar más relacionados con variables ambientales y procesos hormonales, que con la ingesta de $\mathrm{Ca}^{+}$como componente del alimento.

Por otro lado, el alimento concentrado para camarón fue parcialmente consumido por $C$. bocourti; sin embargo, muestra preferencia por el alimento natural, probablemente por- que es más atractivo y aceptado por parte de los ejemplares en el cultivo.

\section{AGRADECIMIENTOS}

Los autores agradecen a la Universidad Simón Bolívar y al departamento administrativo de Ciencia, Tecnología e Innovación (COLCIENCIAS), entidad que financió el proyecto de investigación, en la convocatoria 669-2014. También agradecen al equipo de investigación y personal administrativo de la Universidad Simón Bolívar.

Karen Muñoz-Salas agradece a COLCIENCIAS y a la Gobernación del departamento del Atlántico, por el apoyo recibido en la convocatoria 673 (2014) de formación de capital humano de alto nivel para el departamento del Atlántico, capítulo de maestrías y doctorados nacionales.

\section{BIBLIOGRAFÍA}

Ary, Jr. R. D., Bartell, C. K. \& Poirrier, M. A. (1987). The effects of chelotomy on molting in the blue crab, Callinectes sapidus. J. Shell fish Res., 6, 103-108.

Amador del Ángel, L. E., Lugo-Moreno, J. \& Cabrera-Rodríguez, P. (noviembre, 1992). La quelotomía y la ablación ocular en la obtención de jaiba de concha suave Callinectes sapidus, Rathbun, en condiciones de laboratorio. Ponencia presentada en $45^{\text {th }}$ Proceedings of the Gulf and Caribbean Fisheries Institute. Yucatán, México. 
Amador del Ángel, L. E., Lugo-Moreno, J. \& Cabrera-Rodríguez, P. (1996). La remoción de los quelípedos en la inducción a la ecdisis de la jaiba azul Callinectes sapidus Rathbun en condiciones de laboratorio. Rev. Invest. Mar., 17, 2-3.

Cortés-Jacinto, E. \& Vega-Villasante, F. (2009, enero). La muda y la producción de jaiba suave Baja California Sur. Ponencia presentada en Ciencia, tecnología e innovación para el desarrollo. La Paz, México.

Correa-Daza, J. L. (2002). Determinación y análisis de algunos parámetros biológicos de las jaibas (Callinectes sapidus y Callinectes bocourti), capturadas en la ciénaga de Santa Marta. Informe técnico, documento interno). INPA, $1-41$.

Chang, E. \& Mykles, D. (2011). Regulation of crustaceam molting: A Review and our perspectives. Rev. Gen. Comp. Endocrinol., 172, 323-330. http://dx.doi.org/10.1016/j.ygcen.2011.04.003

Chan, V. T. (2011). Procesos productivos para la obtención de jaiba de concha suave en un sistema cerrado. Foro Iberoam. Rec. Mar. Acui., 3, 137-142.

Charron, L., Geffard, O., Chaumot, A., Coulaud, R., Jaffal, A., Gaillet, V. \& Geffard, A. (2014). Influence of molting and starvation on digestive enzyme activities and energy storage in Gammarus fossarum. Plos one., 9, 1-9. http://dx.doi.org/10.1371/journal. pone.0096393

Ibarra, K. P., Gómez, M. C., Viloria, E. A., Arteaga, E., Cuadrado, I., Martínez M. F., Nieto Y., Rodríguez, J. A., Licero, L. V., Perdomo, L. V., Chávez, S., Romero, J. A. \& Rueda, M. (2014). Monitoreo de las condiciones ambientales y los cambios estructurales y funcionales de las comunidades vegetales y de los recursos pesqueros durante la rehabilitación de la Ciénaga Grande de Santa Marta. Informe técnico. Santa Marta, Colombia: INVEMAR.

Ibarra-Silva, L. I. \& Santamaría-Gallegos, N. A. (1993). Estudios para el semicultivo de jaiba y pescado blanco. Tesis no publicada, Universidad Autónoma, Metropolitana-Unidad de Iztlapalapa, México.

Ibarra, L. E., Olivas, E., Partida, A. L. \& Paredes, D. (2015). Generation of Added Value through the Process of Soft Shell Crab: A Sustainable Development Option in the Coastal Region of Sonora. J. Manage. Sus., 5(2), 57. http://dx.doi.org/10.1371/journal. pone.0096393.10.5539/jms.v5n2p57

Jiménez, E., Pertuz, M., Silva, A. \& Vence, A. (2002). Gestión y evaluación de proyectos: Memoria de aprobación del proyecto $(M A P)$. Universidad del Magdalena, Santa Marta.

Fernández-Luna, I. (1998). Enzimas Digestivas, Crecimiento y Acuicultura de Camarones Penaeus schmitti y Penaeus notialis. Tesis no publicada. Museo Nacional de Historia Natural, Francia.

Fernández-Luna, I., Chávez, M., Preciado, M., Oliva, M., López-López, S., Nolasco, H. \& Viga-Villasante, F. (1999). Contribution to the knowledge on growth and molting of the crab Callinectes arcuatus Ordway (1863) in Nayarit, Mexico. Rev. Invest. Mar., 20, 1-4.

Lasso-Guzmán, N. D. \& Ordóñez-Pardo, J. V. (1987). Contribución al estudio biológico y ecológico de las jaibas (Género Callinectes, Stimpson) de la ciénaga Grande de Santa Marta, Caribe Colombiana. Crustacea: Decapoda: Portunidae. Tesis no publicada. Universidad Nacional de Colombia. 
Lee, K. J., Watson, R. D. \& Roer, R. D. (1998). Molt-inhibiting hormone mRNA levels and ecdysteroid titer during a molt cycle of the blue crab, Callinectes sapidus. Rev. Biochem. Bioph. Res. Co., 249(3), 624-627.

Lozano-Beltrán, G., Reátiga-Aguilar, P., Alarcón-Peña, I. \& Pacheco-Orozco, L. (noviembre, 2015). Identificación de señales de ecdisis en la jaiba roja, (Callinectes bocourti, Milne-Edward, 1879). Ponencia presentada en $68^{\text {th }}$ Proceedings of the Gulf and Caribbean Fisheries Institute. Panamá.

Lozano-Beltrán, G. \& De León-Martínez, G. (2008). Análisis de los desembarques de jaiba en la planta de la pesquera LAROSA del Mar S. A. Barranquilla, Colombia: Pesquera LAROSA del Mar Ltda.

Morillo, N. (2006). Manual de procedimiento industrial del cangrejo azul. Maracay Venezuela: Instituto Nacional de Investigaciones Agrícolas.

Maya de La Cruz, E., Chan-Vadillo, T., GómezMendoza, G. \& Arzola-González, J. (2007). Procesos productivos de la jaiba suave (Callinectes sapidus). Industria Acuícola, 3(4), 19-21

Osterling, M. J. (1995). Manual for Handling and Shedding Blue Crabs (Callinectes sapidus). Virginia, EE. UU.: Institute of Marine Science.

Pérez-González, J. E. (2005). Influencia de la salinidad y el alimento en el proceso de ecdisis dela jaiba verde Callinectes bellicosus (Stmpson, 1859) en un sistema de recirculación. Tesis no publicada. Instituto Politécnico Nacional, México.

Reyes, R., Siam, C. \& Jiménez, E. (2009). La jaiba suave, estudio de factibilidad para una pesquería sostenible. Rev. ACPA., 3, 38-40.

Reyes-Canino, R., Siam, C. \& Jiménez-Hurtado, E. (junio, 2003). La producción de jaiba suave y su incidencia en la sostenibilidad de la biodiversidad en la costa norte de Villa Clara. Ponencia presentada en el XIII Forum de Ciencia y Técnica. Centro de Investigaciones Pesqueras. La Habana, Cuba.

Schleske-Morales, I. C. (2008). El semicultivo de jaiba (Callinectes sp.) como alternativa de inversión dentro de las explotaciones pecuarias. Tesis no publicada, Universidad Veracruzana, México.

Sánchez-Páez, H., Álvarez-León, R., Pinto-Nolla, F., Sánchez-Alférez, A., Pino-Rengifo, J. C., García-Hansen, I. \& Acosta-Peñalosa, M. T. (1997). Diagnóstico y zonificación preliminar de los manglares del Caribe de Colombia. Santa Fe de Bogotá, Colombia: Ministerio del Medio Ambiente.

Siam, C. \& Castelo, R. (2011). Reconocimiento de la premuda en la jaiba azul, $\mathrm{Ca}$ llinectes sapidus, Rathbun (1896). Rev. ACPA., 1, 47-49.

Stagg, C. \& Whilden, M. (1997). Perspectiva histórica de la pesquería y del manejo del cangrejo azul (Callinectes sapi$d u s)$ : En la Bahía de Chesapeake. Rev. Inv. Mar., 25, 93-104.

Valencia-Cuellar, M. J. (1994). Aspectos de la dinámica poblacional de jaibas Callinectes sapidus y C. bocourti de la Ciénaga Grande de Santa Marta, Caribe colombiano (Crustacea: Decapoda: Portunidae) Tesis no publicada, Universidad Javeriana, Colombia.

Valencia-Cuellar, M. J. \& Campos-Campos, N. (1994). Aspectos biológicos de las jaibas Callinectes sapidus y C. bocourti de la Ciénaga Grande de Santa Marta, Caribe colombiano (Crustacea: Decapoda: Portunidae). Rev. Acad. Col. Cienc. Exact. Fis. Nat., 19(75), 730-740.

Vega-Villasante, F., Fernández, I., Preciado, R. M., Oliva, M., Tovar, D. \& Nolasco, 
H. (1999). The activity of digestive enzymes during the molting stages of the arched swimming Callinectes arcuatus Ordway, 1863 (Crustacea: Decapoda: Portunidae). Bull. Mar. Sc., 65(1), 1-9.

Vega-Villasante, F., Cortés, J. E., Reyes, J. A. \& Cupul. M. F. (2006). Manual técnico para la producción de jaiba suave en el pacifico mexicano. Guadalajara, México: Universidad de Guadalajara.
Williams, A. B. (1974). The swimming crabs of the genus Callinectes (Decapoda Portunidae). Fish. Bull., 70(3), 685-798.

Zheng-Junying, L., Chi-Ying, L. \& Douglas-Watson, W. R. (2006). Molecular cloning of a putative receptor guanylyl cyclase from Y-organs of the blue crab, Callinectes sapidus. Rev. Gen. Comp. Endocrinol., 143, 329-336. 
